\title{
ANDES

www.scielo.cl

\section{Trastornos de la conducta alimentaria y obesidad en adolescentes: Otro desafío de nuestros tiempos}

\author{
Eating disorders and obesity in adolescents: Another challenge of our times
}

\author{
Rosa Behara , Verónica Marín ${ }^{\mathrm{b}}$
}

aDepartamento de Psiquiatría, Facultad de Medicina, Universidad de Valparaíso. Valparaíso, Chile

bervicio de Pediatría, Clínica Ciudad del Mar. Viña del Mar, Chile

Recibido: 30 de noviembre de 2020; Aceptado: 15 de abril de 2021

¿Qué se sabe del tema que trata este estudio?

En la adolescencia, debido a los cambios biopsicosociales, se favorece el riesgo para desarrollar un trastorno alimentario. Los pacientes con sobrepeso/obesidad, generalmente no son diagnosticados apropiadamente, ignorándose con frecuencia la significativa superposición entre ambas patologías, con las consiguientes complicaciones evolutivas.
¿Qué aporta este estudio a lo ya conocido?

Esta revisión avanza en la comprensión de las complejas asociaciones entre la obesidad y los desórdenes alimentarios y la perentoria necesidad de exploración de estas temáticas, colocando énfasis en la estrecha e imprescindible contribución entre los profesionales en los ámbitos de salud mental y nutricional.

\section{Resumen}

La obesidad (OB) y los trastornos alimentarios y de la ingestión de alimentos (TAI) son patologías complejas y prevalentes en adolescentes. Se ha demostrado que la OB es un factor de riesgo para el trastorno por atracón y bulimia nerviosa, y viceversa, también los TAI desarrollan OB. Es importante considerar que los adolescentes obesos pueden exhibir criterios atípicos o sub-umbrales de TAI. El objetivo de la presente revisión es describir la relación epidemiológica, clínica y terapéutica entre TAI y $\mathrm{OB}$ en adolescentes, con énfasis en la urgente necesidad de investigación y colaboración entre los profesionales de los ámbitos de salud mental y nutricional-

\begin{abstract}
Obesity $(\mathrm{OB})$ and feeding and eating disorders (FED) are complex and prevalent pathologies in adolescents. $\mathrm{OB}$ has been shown to be a risk factor for developing binge eating disorder and bulimia nervosa, and vice versa, these FED also develop OB. However, obese adolescents may present atypical or sub-threshold criteria for FED. The objective of this review is to describe the epidemiological, clinical, and therapeutic relationship between FED and OB in adolescents, with emphasis on the urgent need for research and collaboration among professionals in the fields of mental health and nutrition.
\end{abstract}

Palabras clave:

Obesidad;

Trastornos

Alimentarios y de la

Ingestión de Alimentos;

Adolescente;

Cirugía Bariátrica

\section{Keywords:}

Obesity;

Feeding and Eating

Disorders;

Adolescent;

Bariatric Surgery 


\section{Introducción}

La obesidad (OB) y los trastornos alimentarios y de la ingestión de alimentos (TAI) son patologías complejas y prevalentes ${ }^{1-3}$. Pese a que ambas comparten aspectos etiopatogénicos biopsicosociales, generalmente son abordadas separada y parcialmente, desconociéndose una significativa co-ocurrencia entre ellas ${ }^{4-6}$.

La restricción dietética, la preocupación por el peso y figura son frecuentes en jóvenes con sobrepeso/OB, previo al inicio de su tratamiento ${ }^{7}$, siendo más habituales en ellos conductas poco saludables de control ponderal ${ }^{8}$, constituyendo factores de riesgo para desarrollar un TAI; pero, que por tratarse de pacientes con sobrepeso/OB, generalmente no son diagnosticados apropiadamente y los programas de salud pública, con frecuencia han ignorado la significativa superposición entre la OB y los TAI.

El objetivo de este artículo es describir la relación epidemiológica, clínica y terapéutica entre los TAI y la $\mathrm{OB}$ en adolescentes y proponer estrategias de prevención y tratamiento conjunto.

\section{Aspectos epidemiológicos}

En América Latina, los porcentajes de trastorno por atracones en obesos de 14 a 52,9 años, que acuden a programas para perder peso, varía entre $16 \%$ a $51,6 \%{ }^{9}$. En estadounidenses adultos fluctúan entre $4 \%$ a $49 \%{ }^{10}$ y en adolescentes entre $15,4 \%$ a $41,7 \%{ }^{11}$. Además, la velocidad en que aumentan ambas patologías juntas es significativamente mayor ( 4,5 veces) que el incremento en personas con OB sola ( 1,6 veces) o con TAI sin OB ( 3,1 veces $)^{12}$.

En Chile, un estudio con adolescentes obesos que ingresaron a tratamiento, reportó que $19,6 \%$ de las mujeres y $12,2 \%$ de los hombres presentaron TAI, siendo mayor en aquellos con índice de masa corporal más elevado $(20,5 \%)^{13}$.

\section{Obesidad y trastornos alimentarios}

Los adolescentes obesos suelen presentar criterios atípicos o sub-umbrales de TAI, debido al exceso de peso corporal y a pesar de una importante baja ponderal, al no alcanzar un estado de desnutrición, se retrasan su diagnóstico y tratamiento ${ }^{14}$.

Meierer et al. ${ }^{15}$ verificaron que en adolescentes con anorexia nerviosa e historia de sobrepeso, la baja ponderal era mucho mayor que en aquellos que no presentaban antecedente de sobrepeso ( 7 versus 3,8 puntos). Asimismo, demostraron que más de un tercio presentaba este antecedente, generando una tardanza entre el reconocimiento de la anorexia nerviosa y su cabal diagnóstico. Kennedy et al. ${ }^{16}$ observaron un mayor porcentaje de pérdida ponderal ( $27,4 \%$ versus $16,2 \%)$, en pacientes con anorexia nerviosa típica o atípica, con sobrepeso/OB pre-mórbidos y una duración de la enfermedad más prolongada que los pacientes sin tal historial.

Igualmente, se debe destacar que el trastorno por atracones es el TAI más frecuente asociado con sobrepeso/OB, encontrándose entre $10 \%$ a $25 \%$ de los pacientes $^{17,18}$, por lo que su detección sistemática es fundamental.

\section{Hábitos alimentarios}

He et al. ${ }^{19}$ estimaron que la prevalencia de atracones y descontrol en el comer se presentan en $22,2 \%$ y $31,2 \%$ de los niños y adolescentes con sobrepeso/OB, respectivamente.

La adicción a la comida se ha asociado con una mayor psicopatología en personas con TAI y OB, distinguiéndose tres grupos: 1) disfuncional (mayor prevalencia de otros TAI y bulimia nerviosa, mayor gravedad de la patología alimentaria y más rasgos disfuncionales de personalidad); 2) moderado (alta prevalencia de bulimia nerviosa, trastorno de personalidad y niveles moderados de psicopatología alimentaria); 3) adaptativo (alta prevalencia de $\mathrm{OB}$, trastorno por atracones, bajos niveles de psicopatología alimentaria y rasgos de personalidad más funcionales $)^{20}$.

Para Stojek et al. ${ }^{21}$, los adolescentes con un patrón alimentario descontrolado emocional, representan un subconjunto, con un riesgo especialmente alto de exacerbación del TAI y aumento ponderal excesivo. Rose et al. ${ }^{22}$ comprobaron que los adolescentes con $\mathrm{OB}$ severa, con más dificultades en el control impulsivo durante estados de ánimo negativos, presentaron mayormente una alimentación de tipo emocional y adicción a la comida, asociadas con una menor calidad de vida. Las intervenciones destinadas a reducir los afectos negativos, la impulsividad, y a mejorar las habilidades de afrontamiento no basadas en la alimentación, pueden contribuir a optimizar la calidad de vida y ameritan un estudio a fondo.

Kass et al. ${ }^{23}$, comprobaron que los jóvenes con sobrepeso/OB que se alimentaban en secreto, eran más propensos a la depresión, a la restricción dietética, a atracones y purgas, presagiando un mayor riesgo de aumento ponderal y de TAI.

\section{Predictores de riesgo}

Los TAI y la OB comparten factores de riesgo biológicos, ambientales, comportamientos y fenotipos neuro-cognitivos intermedios. $\mathrm{La} \mathrm{OB}$ es un factor de riesgo específico, tanto para el trastorno por atracón y bulimia nerviosa y las personas con estos TAI a menudo también tienen $\mathrm{OB}^{24}$. Una controvertida teoría postula que ambos desórdenes pertenecen a un mismo espectro de trastornos relacionados con la alimentación y el peso ${ }^{25}$. Pacientes obesos con TAI co-mórbi- 
dos, principalmente trastorno por atracones, muestran más capacidad para $\operatorname{comer}^{26}$, psicopatología general $^{27}$ y de la personalidad ${ }^{28}$, aspectos considerados predisponentes para desencadenar un TAI en personas con sobrepeso/OB.

Los comentarios acerca del peso y dieta en familia, aunque sean bien intencionados, pueden percibirse como hirientes por el adolescente y contribuir al surgimiento de ambos cuadros ${ }^{29-31}$. Tanto las burlas familiares, como la insatisfacción corporal, también predicen el desarrollo de sobrepeso, atracones y conductas inadecuadas de control ponderal en los niños ${ }^{32,33}$.

Un análisis desde el nacimiento, determinó que la alimentación materna desordenada, su insatisfacción corporal e historial de sobrepeso, predijeron una aparición de ingestión secreta en el niño, durante los primeros 5 años de vida, que aunque no es equivalente a un trastorno por atracón, consiste en comer en solitario debido a la vergüenza que el descontrol provoca, por lo que se plantea como un indicador de riesgo ${ }^{34}$.

El abuso infantil, es otro predisponente para ambas patologías. Las víctimas pueden recurrir a los alimentos para aliviar el estrés, manejar el trauma, bloquear sentimientos y emociones no deseadas o expresar odio hacia el propio cuerpo ${ }^{35}$. También cumpliría un rol protector, ya que los niños obesos serían menos atractivos para un potencial abusador ${ }^{36}$. En las adolescentes femeninas, el abuso se ha asociado con depresión, TAI y baja autoestima. Algunos sobrevivientes de abuso sexual luchan por adelgazar en un intento de negar la propia sexualidad. Otros se obsesionan con hacer dieta o purgar para lograr la perfección corporal, sentirse más poderosos, invulnerables y recuperar la autoestima ${ }^{37}$. Los atracones, las purgas y el hambre, implicarían un autocastigo para mitigar su culpa. En consecuencia, la posibilidad de abuso sexual debe ser evaluada y su dato incluido regularmente en el estudio de pacientes con TAI y/o sobrepeso/OB, especialmente en el género femenino ${ }^{38}$.

\section{Obesidad, trastornos alimentarios, cirugía bariátrica y evolución post-quirúrgica}

En adolescentes, el tratamiento médico de la $\mathrm{OB}$ es el de elección, pero en cuadros graves acompañados de comorbilidad, que no responden, la cirugía bariátrica es una opción ${ }^{39}$. Sin embargo, no todos se beneficiarían, por lo que la selección, preparación para un cambio del estilo de vida, y seguimiento pre y postoperatorio a largo plazo, son claves

Individuos que buscan cirugía bariátrica, habitualmente muestran tasas elevadas de atracones y otros $\mathrm{TAI}^{40}$. Aunque el consumo de cantidades objetivamente grandes de alimentos es difícil post-cirugía, aquellos que continúan presentando descontrol alimentario, experimentan pérdida de peso sub-óptima, o inclusive, recuperación ponderal. Actualmente, no existen estudios concluyentes acerca de predictores de éxito post cirugía, pero los pacientes con trastornos por atracones podrían beneficiarse, agregando una intervención de regulación de las emociones en el período pre y postoperatorio ${ }^{41}$. Prácticamente no se registran casos de anorexia nerviosa en el pre-operatorio, pero si cierta evidencia para su desarrollo post-quirúrgico ${ }^{42-44}$. La bulimia nerviosa, es relativamente poco frecuente previa a la cirugía, alcanzando el $3 \%$, y se dispone de escasos datos sobre cambios post-cirugía. Una razón probable puede ser su sub-reporte por temor a no ser elegibles para este procedimiento ${ }^{10}$. El trastorno por atracones es el más común antes de la cirugía, con tasas entre $4 \%$ a $49 \%{ }^{11}$ en adultos y $15,4 \%$ y $41,7 \%$, en adolescentes; constatándose su disminución post-quirúrgica, a corto plazo, en todas las edades ${ }^{41}$. En un seguimiento nacional, 3 años post bypass gástrico en adultos, se observó que no había diferencia en la frecuencia del trastorno por atracones entre pacientes operados versus no operados. También se constató una baja calidad de vida post-cirugía, en cuanto a salud mental, asociada particularmente al ánimo y a la sexualidad ${ }^{42}$.

Se han demostrado mejoras iniciales del comer descontrolado a 7 años de sondeo posterior al bypass gástrico o banda gástrica, pero la proporción de pacientes con trastorno por atracón a largo plazo, aumenta. Un $4,8 \%$ informó la aparición de trastorno por atracón que no tenía previamente, $3,8 \%$ reaparición de este cuadro pre-existente y $9,2 \%$ remitió, si bien, en este grupo, $46,6 \%$ mantuvo un comer descontrolado, lo que puede conllevar un resultado quirúrgico negativo o sub-óptimo ${ }^{41}$.

Otro análisis post-cirugía a 5 años, constató que los problemas de salud mental persisten en los adolescentes, a pesar de la considerable pérdida de peso. Así, mientras ésta puede mejorar muchos parámetros físicos, el alivio de las complicaciones psicológicas no es claro, y un equipo bariátrico debe ofrecer apoyo a largo plazo posterior a la intervención ${ }^{40}$.

\section{Prevención y tratamiento conjunto de ambas patologías}

Comprender el conocimiento y las creencias de las partes interesadas en la prevención y tratamiento para OB y TAI, es crucial para desarrollar intervenciones eficaces. Además, realizar esfuerzos integrados de promoción de la salud que se centran en el riesgo compartido (baja autoestima e insatisfacción corporal) y se apoyen en factores protectores (alimentación saludable y ejercicio regular), dirigidos a niños pequeños, adolescentes y padres, pueden tener resultados positi$\operatorname{vos}^{5}$.

La mayoría de los adolescentes que padecen un TAI no presentan $\mathrm{OB}$ previamente, pero pueden surgir en 
algunos pacientes obesos, en un intento por perder peso. La atención debe centrarse en un estilo de vida saludable más que en el peso. La información disponible sugiere que la prevención y el tratamiento de la $\mathrm{OB}$, realizados correctamente, no predisponen a los $\mathrm{TAI}^{41}$, demostrándose incluso que el tratamiento de la $\mathrm{OB}$, estructurado y administrado profesionalmente, se asocia con una reducción de la prevalencia, el riesgo y los síntomas de TAI La evidencia reciente muestra que las terapias familiar y cognitivo conductual para la OB infanto-juvenil, son efectivas en reducir el peso, las preocupaciones sobre la figura y desalentar los $\mathrm{TAI}^{42,43}$.

\section{Análisis y conclusiones}

Se resalta la importancia de un abordaje multidisciplinario, fundamentalmente desde el ámbito de la salud mental y nutricional, y un diagnóstico conjunto de atracones, trastornos emocionales y de personalidad en adolescentes obesos, para una mejor prevención y desenlace de ambas patologías. La necesidad de incluir la detección temprana del TAI (especialmente el trastorno por atracón), junto a la ejecución de programas terapéuticos integrados, son críticos para una mejor evolución. Los profesionales de la salud tienden a no identificarlos en personas obesas, y sus síntomas son más difíciles de reconocer si no se indagan rutinaria y dirigidamente.

Si la prevención y tratamiento de la OB se realizan correctamente, ésta no predispone a los $\mathrm{TAI}^{42}$; al contrario, tales programas, que incluyen a la familia, muestran una reducción en la utilización de vómitos auto-inducidos y otras medidas compensatorias patológicas para controlar el peso ${ }^{43}$.

Otros enfoques basados en la experiencia clínica, animan a los padres a incluir mayor disponibilidad de alimentos sanos y beneficiosos, menos tiempo de pantallas, más comidas caseras familiares, con menos distracciones y conversaciones sobre el peso, la figura y la dieta, pueden promover una imagen corporal saludable ${ }^{41}$.

En adolescentes, el tratamiento médico de la $\mathrm{OB}$ es el recomendado, pero en casos severos en los cuales co-existen complicaciones médicas graves, que no responden adecuadamente a las intervenciones de cambio en el estilo de vida, pueden verse beneficiados con tratamiento quirúrgico. No obstante, no todos se verían favorecidos por éste, por lo que su elección y controles pre y post quirúrgicos a largo plazo, son esenciales para obtener buenos resultados terapéuti$\cos ^{52}$. Si un adolescente obeso ha requerido llegar a someterse a una operación quirúrgica, es indispensable considerar los principios y fundamentos del "Consenso chileno de salud mental para pacientes sometidos a cirugía bariátrica" ${ }^{53}$, orientado a lograr el éxito de la intervención.

\section{Conflicto de intereses}

Las autoras declaran no tener conflicto de intereses.

\section{Referencias}

1. Olivares S, Zacarías I. Estudio para revisión y actualización de las guías alimentarias para la población chilena. Santiago de Chile: Gobierno de Chile, Ministerio de Salud. Instituto de Nutrición y Tecnología de los Alimentos (INTA), Universidad de Chile; 2013. Disponible en: http://web.minsal.cl/ alimentos_nutricion.

2. Vio F. Aumento de la obesidad en Chile y el mundo. Rev Chil Nutr. 2018;45(1):6-6.

3. JUNAEB. Mapa Nutricional de la Junta Nacional de Auxilio Escolar y Becas. https://www.junaeb.cl/ wp-content/uploads/2013/03/MapaNutricional-2019-1.pdf

4. Q da Luz F, Hay P, Touyz S, et al. Obesity with comorbid eating disorders: Associated health risks and treatment approaches. Nutrients. 2018;10(7):829.

5. Bullivant B, Denham A, Stephens C, et al. Elucidating knowledge and beliefs about obesity and eating disorders among key stakeholders: Paving the way for an integrated approach to health promotion. BMC Public Health. 2019;19(1):1681-91.

6. Hay P, Mitchison D. Eating disorders and obesity: The challenge for our times. Nutrients. 2019;11:1055-59.

7. Goldschmidt A, Aspen V, Sinton M, et al. Disordered eating attitudes and behaviours in overweight youth. Obesity. 2008;16(2):257-64.

8. Lampard A, Maclehose R, Eisenberg M. Adolescents who engage exclusively in healthy weight control behaviours: Who are they? Int J Behav Nutr Phys Act. 2016;13:5-9.

9. Palavras M, Kaio G, Mari J, et al. A review of Latin American studies on binge eating disorder. Braz J Psychiatry. 2011;33:81-94.

10. Opolski M, Chur-Hansen A, Wittert G. The eating-related behaviours, disorders and expectations of candidates for bariatric surgery. Clin Obes. 2015;5(4):165-97.

11. Carriere C, Michel G, Féart C, et al.
Relationships between emotional disorders, personality dimensions, and binge eating disorder in French obese adolescents. Arch Pediatr. 2019;26(3):13844.

12. Darby A, Hay P, Mond J, et al. The rising prevalence of comorbid obesity and eating disorder behaviours from 1995 to 2005 . Int J Eat Disord. 2009;42(2):104-8.

13. Marín V, Sommer K, Agurto P, et al. Eating disorders risk among obese adolescents Rev Med Chil. 2014;142(10):1253-8.

14. Taylor S, Ditch S, Hansen S. Identifying and preventing eating disorders in adolescent patients with obesity. Pediatr Ann. 2018;47(6):232-7.

15. Meierer K, Hudon A, Sznajder M, et al. Anorexia nervosa in adolescents: Evolution of weight history and impact of excess premorbid weight. Eur J Pediatr. 2019;178(2):213-9.

16. Kennedy G, Forman S, Woods E, et al. History of overweight/obesity as predictor of care received at 1-year follow-up in 
adolescents with anorexia nervosa or atypical anorexia nervosa. J Adolesc Health. 2017;60(6):674-9.

17. McCuen-Wurst C, Ruggieri M, Allison $\mathrm{K}$. Disordered eating and obesity: Associations between binge eatingdisorder, night-eating syndrome, and weight-related comorbidities. Ann N Y Acad Sci. 2018;1411(1):96-105.

18. Bohon C. Binge eating disorder in children and adolescents. Child Adolesc Psychiatric Clin N Am. 2019;28:549-55.

19. He J, Cai Z, Fan X. Prevalence of binge and loss of control eating among children and adolescents with overweight and obesity: An exploratory meta-analysis. Int J Eat Disord. 2017;50(2):91-103.

20. Jiménez-Murcia S, Agüera Z, Paslakis G, et al. Food addiction in eating disorders and obesity: analysis of clusters and implications for treatment. Nutrients. 2019;11(11):2633.

21. Stojek M, Tanofsky-Kraff M, Shomaker $\mathrm{L}$, et al. Associations of adolescent emotional and loss of control eating with 1-year changes in disordered eating, weight, and adiposity. Int J Eat Disord. 2017;50(5):551-60.

22. Rose M, Nadler E, Mackey E. Impulse control in negative mood states, emotional eating, and food addiction are associated with lower quality of life in adolescents with severe obesity. J Pediatr Psychol. 2018;43(4):443-51.

23. Kass A, Wilfley D, Eddy K, et al. Secretive eating among youth with overweight or obesity. Appetite. 2017;114:275-81.

24. Villarejo C, Fernández-Aranda F, Jiménez-Murcia $S$, et al. Lifetime obesity in patients with eating disorders: increasing prevalence, clinical and personality correlates. Eur Eat Disord Rev. 2012;20(3):250-4.

25. Wilson G. Eating disorders, obesity and addiction. Eur Eat Disord Rev. 2010;18:341-51.

26. Fassino S, Leombruni P, Piero A, et al. Mood, eating attitudes, and anger in obese women with and without binge eating disorder. J Psychosom Res. 2003;54:55966.

27. Zeeck A, Stelzer N, Linster HW, et al. Emotion and eating in binge eating disorder and obesity. Eur Eat Disord Rev. 2011;19(5):426-37.

28. Nasser J, Gluck M, Geliebter A. Impulsivity and test meal intake in obese binge eating women. Appetite. 2004;43:303-7.

29. Berge J, MacLehose R, Loth K, et al. Parent-adolescent conversations about eating, physical activity and weight: Prevalence across sociodemographic characteristics and associations with adolescent weight and weight-related behaviours. J Behav Med. 2015;38(1):12235.

30. Loth K, Neumark-Sztainer D, Croll J. Informing family approaches to eating disorder prevention: perspectives of those who have been there. Int J Eat Disord. 2009;42(2):146-52.

31. Berge J, Maclehose R, Loth K, et al. Parent conversations about healthful eating and weight: Associations with adolescent disordered eating behaviours. JAMA Pediatr. 2013;167(8):746-53.

32. Eisenberg M, Berge J, Fulkerson JA, et al. Associations between hurtful weight-related comments by family and significant other and the development of disordered eating behaviours in young adults. J Behav Med. 2012;35(5):500-8

33. Neumark-Sztainer D, Paxton S, Hannan $\mathrm{P}$, et al. Does body satisfaction matter? Five-year longitudinal associations between body satisfaction and health behaviours in adolescent females and males. J Adolesc Health. 2006;39(2):24451.

34. Stice E, Agras W, Hammer L. Risk factors for the emergence of childhood eating disturbances: a five-year prospective study. Int J Eat Disord. 1999;25(4):375-87.

35. Behar R, Arancibia M, Sepúlveda E, et al. Chapter 9. Child sexual abuse as a risk factor in eating disorders. In: Morton $\mathrm{N}$, editor. Eating disorders in the 21st century. Eating disorders: Prevalence, risk factors and treatment options. New York: Nova Science Publishers. 2016;149-72. ISBN: 978-1-53610-062-4.

36. Ackard D, Neumark-Sztainer D, Hannan $\mathrm{P}$, et al. Binge and purge behaviour among adolescents. Child Abuse \& Neglect. 2001;25(6):771-85

37. Rayworth BB, Wise LA, Harlow BL. Childhood abuse and risk of eating disorders in women. Epidemiology. 2004;15(3):271-8.

38. Opydo-Szymaczek J, Jarząbek-Bielecka G, Kędzia W, et al. Child sexual abuse as an etiological factor of overweight and eating disorders - considerations for primary health care providers. Ginekol Pol 2018;89(1):48-54.

39. Paulus G, de Vaan L, Verdam F, et al. Bariatric surgery in morbidly obese adolescents: a systematic review and meta-analysis. Obes Surg. 2015;25(5):86078.

40. Childerhose J, Alsamawi A, Mehta T, et al. Adolescent bariatric surgery: A systematic review of recommendation documents. Surg Obes Relat Dis. 2017;13(10):1768-79.
41. Benzerouk F, Djerada Z, Bertin E, et al. Contributions of emotional overload, emotion dysregulation, and impulsivity to eating patterns in obese patients with binge eating disorder and seeking bariatric surgery. Nutrients. 2020;12:3099-114.

42. American Psychiatric Association. Diagnostic and statistical manual of mental disorders. Fifth Edition (DSM-5). Washington, DC: American Psychiatric Association; 2013.

43. Deitel M. Anorexia nervosa following bariatric surgery. Obes Surg. 2002;12:72930.

44. Conceição E, Orcutt M, Mitchell J, et al. Eating disorders after bariatric surgery: A case series. Int J Eat Disord. 2013;46:2749.

45. Järvholm K, Bruze G, Peltonen M, et al. 5 -year mental health and eating pattern outcomes following bariatric surgery in adolescents: A prospective cohort study. Lancet Child Adolesc Health. 2020;4(3):210-9.

46. Leiva M, Fuentealba C, Boggiano C, et al. Calidad de vida en pacientes operadas de bypass gástrico hace más de un año: Influencia del nivel socioeconómico. Rev Med Chil. 2009;137:625-33.

47. Smith K, Orcutt M, Steffen K, et al. Loss of control eating and binge eating in the seven years following bariatric surgery. Obes Surg. 2019;29(6):1773-80.

48. Golden N, Schneider M, Wood C, et al. Preventing obesity and eating disorders in adolescents. Pediatrics. 2016;138(3):20161649.

49. Jebeile H, Gow M, Baur L, et al. Treatment of obesity, with a dietary component, and eating disorder risk in children and adolescents: A systematic review with meta analysis. Obesity Reviews 2019;20:1287-98.

50. Hayes J, Fitzsimmons-Craft E, Karam A, et al. Disordered eating attitudes and behaviours in youth with overweight and obesity: Implications for treatment. Curr Obes Rep. 2018;7(3):235-46.

51. Balantekin K, Hayes J, Sheinbein D, et al. Patterns of eating disorder pathology are associated with weight change in familybased behavioural obesity treatment. Obesity. 2017;25(12):2115-22.

52. Stice E, Desjardins C. Interactions between risk factors in the prediction of onset of eating disorders: exploratory hypothesis generating analyses. Behav Res Ther. 2018;105:52-62.

53. Leiva M, Cruz M, Díaz P, et al. Manejo psicológico del paciente sometido a cirugía bariátrica. Consenso Núcleo de Psicólogos de Cirugía de la Obesidad de Chile. Rev Med Chil. 2020;148:518-27. 